\title{
Kinetic Architecture and Foldable Surface
}

\author{
By Vincenzo Sapienza* \\ Gianluca Rodonò $\grave{~}^{\dagger}$
}

In the recent decades, the flexibility of the modern human requirements and the growing need for economic sustainability have increased the interest in architecture with changeable configuration. In this sense, there are many examples defined as interactive or responsive architectures, in which the configuration can adapt to users' requirement or climate changes. In particular, a series of activities, from humanitarian or environmental emergencies to social and cultural events, require lightweight, easily transportable and transformable objects. An answer to these requirements can be provided by the use of a type of mechanism that is defined as "folding". The University of Catania has formed a multidisciplinary research group addressed to develop a project called KREO (Kinetic Responsive Envelope by Origami). The goal is to realize a pre-folded reusable envelope, in composite material. In the present article the first results are shown, related to the analysis of the state of the art, the base material and the possible applications.

\section{Introduction}

The kinetic architectures have antique and noble ancestors, if you consider the shelters of the nomadic people or the velaria of the Romans theatres (Figure 1 ), and other examples in which the building components could be positioned and removed in a short time. To obtain this result, the related architectures had to be designed as mechanisms, to have various possibilities of movement of its parts.

As it is known, this kind of approach had a limited number of applications, up to a large part of the XX century. To confirm this, you can consider the Monument to the Third International, designed by Vladimir Tatlin in 1919, that was never built, despite its great ideological meaning. ${ }^{1}$ Moreover you have to take into account that the architecture has always been considered an artistic form related to eternity. Dante Alighieri underlined this concept by writing the following verses on the top of the Hell Door: Before Me Nothing Was Created That Was Not Eternal, and I Last Eternally. ${ }^{2}$

\footnotetext{
* Associate Professor, University of Catania, Italy.

${ }^{\dagger}$ PhD Candidate, University of Catania, Italy.

1. This building was composed by three overlapped rooms. They had different destinations and rotated at different periods. The monument had to allude to the different roles of the components of the society.

2. Dante Alighieri, Divine Comedy, Inferno, III, 7-8.
} 
Nowadays, the need of social life grows in number and in complexity. To follow them, it is necessary to have flexible, light, reusable service facilities. This kind of units could be useful, for example, for environmental emergencies, for fashion events, for the temporary hospitality of the migrants and for the safety of Cultural Heritage.

The last point is particularly important in the European countries because of their large number of monuments that are exposed to a very high risk, depending on weather, pollution or vandal activities. To mitigate it, the European Community is going to open a call in the Horizon 2020 Program. ${ }^{3}$

To minimize solar radiation, the rain or other dangers, it can be used as a kind of light shelter, to remove as soon as the disturbances are over. This achievement can be obtained by using a particular type of kinetic architecture that is called foldable architecture.

This expression can assume a number of meanings. We refer to a kind of building element that can change their configuration, if they are stressed. Usually the movement is an effect of the shape-memory of the base material that is due to the folding.

Their design is a complex process in which you need various contributions, from the chemistry of the materials to the design, as it will be discussed.

\section{Foldable Architecture}

\section{Typology}

The typological analysis, about what has been already realized, is a fundamental step, to correctly address the future work. In particular we have started from the first approach to this matter that is due to Frei Otto, who tried to improve it, to take under consideration the more recent examples.

Folding (Figure 2) has been indicated as one of the types of movement (like rolling, sliding, rotating, ...) that allows building components or to the whole building system to modify its own configuration. ${ }^{4}$ The construction system, that allows the folding movement, can be either by membranes or by rigid elements. In the first case the flexible surface is constructed with light material, generally supported by a spatial frame structure with hinged elements that allow movements. In the second case the surface is constructed with one or more overlapped materials and is self-supporting. Both systems are constructed with parallel, central, circular or peripheral directions of movement.

A further typological classification, that is useful to consider in our study, divides the realizations of the last years in relation to the needs which kinetic architecture tries to give an answer trough specific requisites. In this sense the most frequently used characteristics are: the intrusion resistance, with

3. See: Innovative solutions for the conservation of $20^{\text {th }}$ century cultural heritage (NMBP 43-2017); the call will be opened at the end of 2016.

4. Frei Otto, ILEK/IL5 (Stuttgart: ILEK press, 1972). 
protection systems for envelops; the flexibility and adaptability of the space; the control of the noise and the acoustic isolation or absorption.

The search aims at cataloging the projects of the last years by following these two typological classifications, to individualize possible future developments on this thematic.

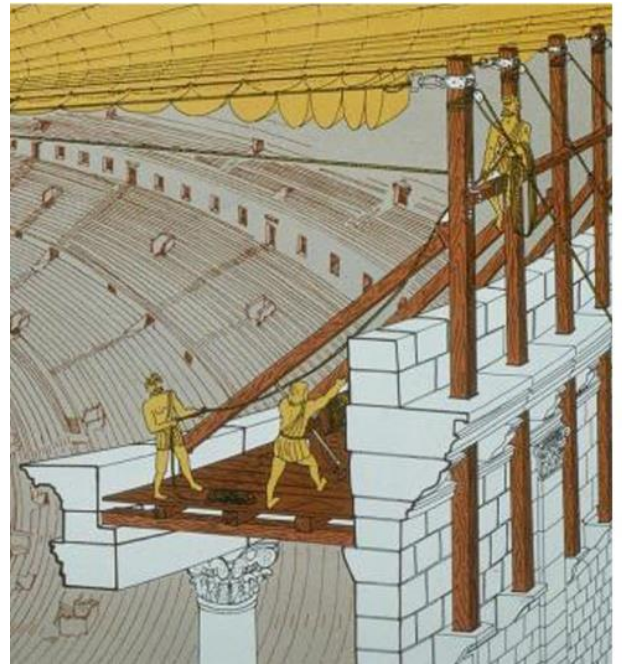

Figure 1. Velarium for Roman

Theater and Anphitheater
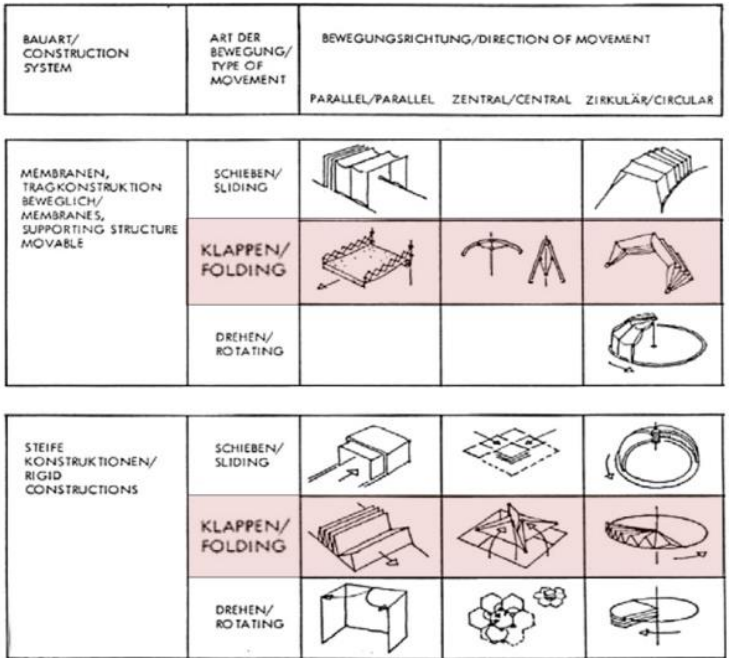

Figure 2. Typological Classification by $F$. Otto. Folding Type is Highlighted

As shown in Table 1, folding is the type of movement mostly used in the practice because of its expressive potential and its propensity to movement. For this reason the research is focused on foldable surfaces (Table 2). 
Vol. 2, No. 3

Sapienza: Kinetic Architecture and Foldable Surface

Table 1. Typolgical Classification of the Recent Kinetic Architecture

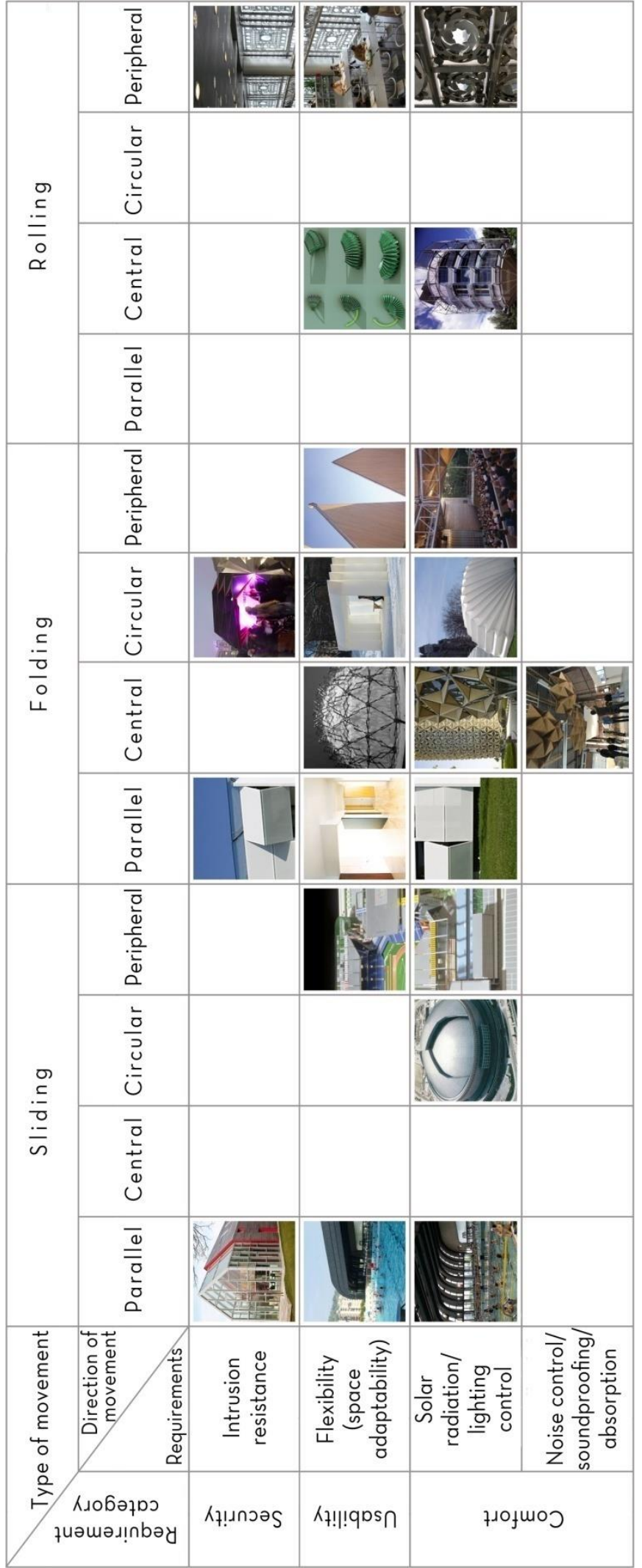


Table 2. Recent Foldable Architecture

\begin{tabular}{|c|c|c|c|c|c|}
\hline \multirow{2}{*}{\multicolumn{2}{|c|}{ 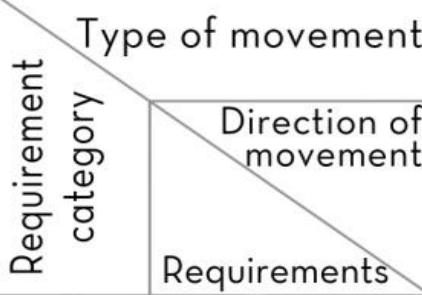 }} & \multicolumn{4}{|c|}{ Folding } \\
\hline & & Parallel & Central & Circular & Peripheral \\
\hline 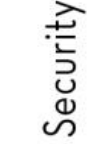 & $\begin{array}{l}\text { Intrusion } \\
\text { resistance }\end{array}$ & & & & \\
\hline $\begin{array}{l}\stackrel{\overrightarrow{ \pm}}{=} \\
\stackrel{\overline{0}}{0} \\
\stackrel{0}{0}\end{array}$ & $\begin{array}{c}\text { Flexibility } \\
\text { (space } \\
\text { adaptability) }\end{array}$ & & & & \\
\hline \multirow{2}{*}{ 蒙 } & $\begin{array}{l}\text { Solar } \\
\text { radiation/ } \\
\text { lighting } \\
\text { control }\end{array}$ & & & & $2-\frac{3}{3}$ \\
\hline & $\begin{array}{c}\text { Noise control/ } \\
\text { soundproofing/ } \\
\text { absorption }\end{array}$ & & In & & \\
\hline
\end{tabular}

This kind of envelope is often used to make temporary structures like the projects of Corogami Hut (Figure 3) and ReCover (Figure 4). The main concept of the projects is to fully exploit the characteristics of a single material (lightness, inexpensiveness and sustainability) by using it as the only building component. In addition, both projects are made of polypropylene elements with geometry and movement inspired by the Japanese art of Origami. The first of them is addressed to realize facilities for an open ice rink and the second one is an emergency shelter for four people.

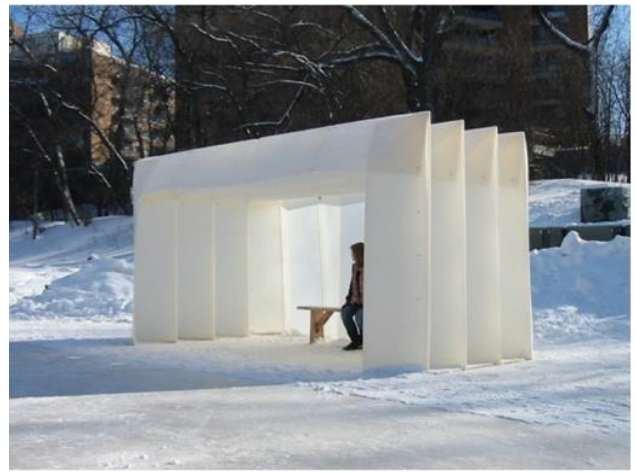

Figure 3. Corogami Hut

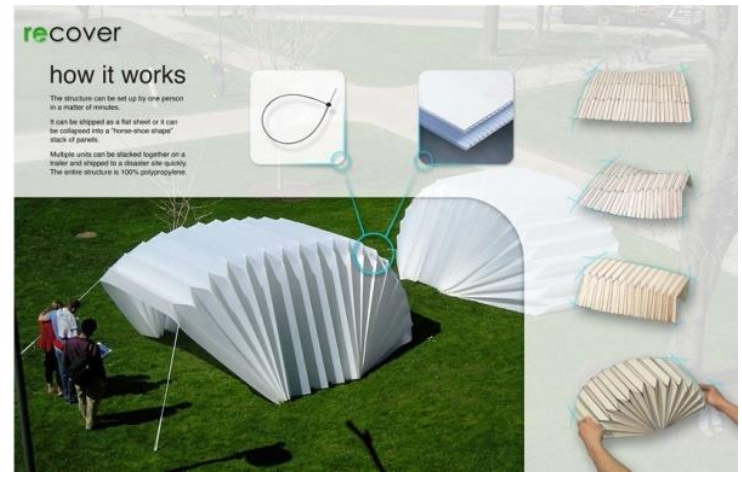

Figure 4. Accordion ReCover Shelter 


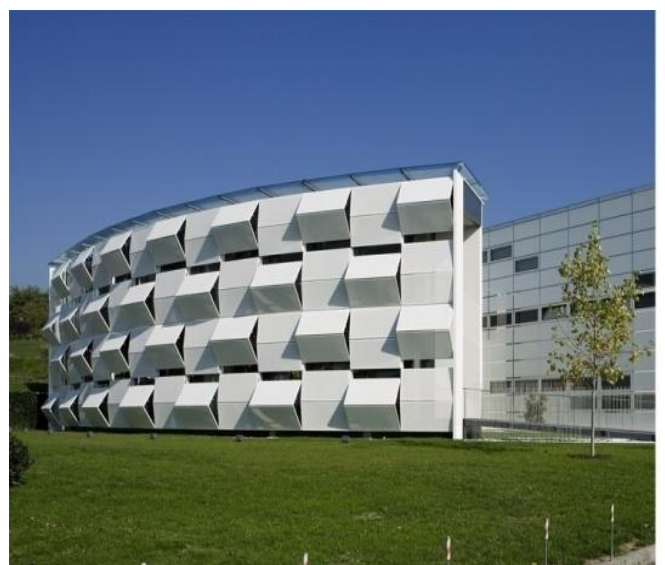

Figure 5. KieferTechnicShowroom

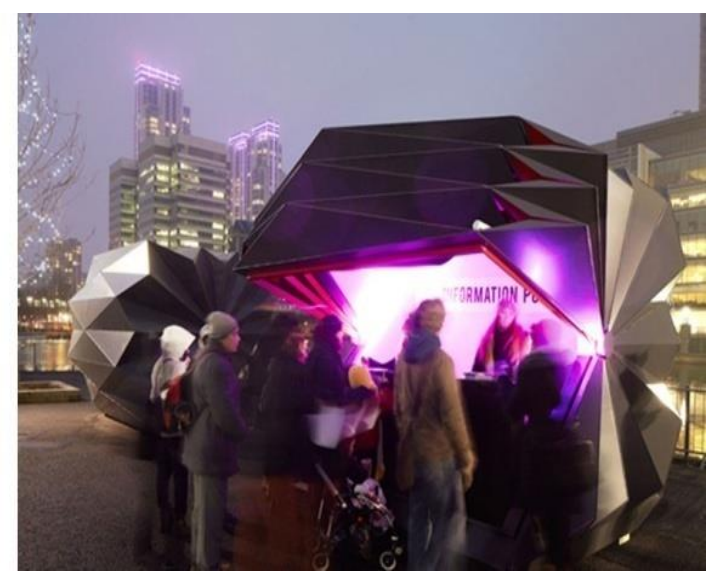

Figure 6. Kiosk

Thanks to the folding ability of the surface they have incredible configuration variability and a great facility of assemblage, so fully answering to the requisite of adaptability.

Another very interesting project is the Kiosk project (Figure 6). It has a similar geometry and movement compared with the previous ones, but in order to answer to the requisite of intrusion resistance, it is made with metallic elements that protect it from vandals.

Intrusion resistance is also the goal of the envelop of the Kiefer Technic Showroom (Figure 5). This is composed by a series of hinge panels with a parallel direction of movement that forms a mutable façade. The goal is also to create a responsive filter for solar radiation by creating a climate adaptive building shell. ${ }^{5}$

One of the most popular projects with an adaptive façade is the Al Bahr Towers (Figures 7 and 8). Inspired by the mashrabiya, the shell is composed by a series of shading units with PTFE (polytetrafluoroethylene) panels controlled by a complex Building Management System (BMS). ${ }^{6}$ Every single unit has a central type of folding movement, like an umbrella, to control direct solar gain, during the day.

5. Roel C. G. M. Loonen et al., "Climate adaptive building shells: State-of-the-art and future challenges," Renewable and Sustainable Energy Reviews 25 (2013): 483-493.

6. A BMS improves the regulation and controls the functioning of appliances, such as luminaires, window blinds and heating equipment in a building. The appliances are connected via a communication bus to a control system, which performs the automatic regulation and control. 

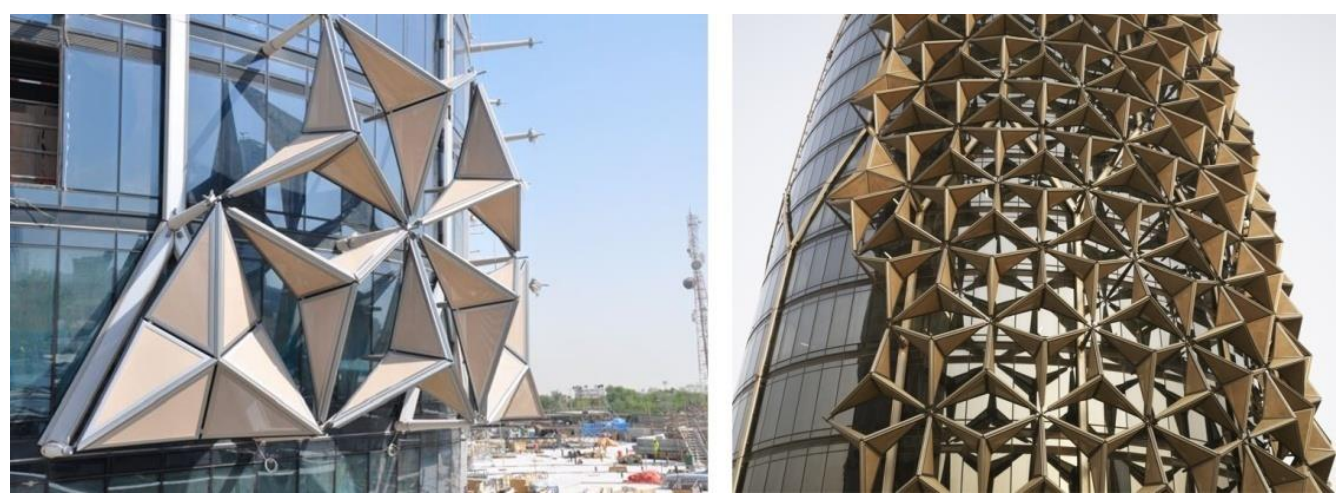

Figures 7 and 8. Al Bahr Towers

\section{Use}

In the last few years, foldable surfaces are becoming popular in architecture and engineering fields because of the characteristics that they are able to perform.

First of all, consideration should be given to mechanical properties of folded plate structures. As it has already been said, making a fold on a surface produces stiffening along the crease. For example a folded surface will be able to cover a much greater space than a plane surface of the same material. The additional strength creates the so called "form-resistant structure". This property is utilized in numerous applications in which it is necessary to optimize the employment of the materials as, for instance, the realization of buildings' coverage, envelopes or pavilions.

The articulation and the charm of the complex geometries, realized through the tessellation, together with the clarity of the distribution of the tensions along the form/structure, represent one of the reasons for the great success of applications of these kinds of components in architecture. Indeed in the 1920s Josef Albers stimulated his students of the preliminary course of the Bauhaus to the realization of models, in paper and in acetate, of folding surfaces, investigating the relationships among material, form and structure. ${ }^{7}$

The folding ability and therefore the kinematic of the surfaces are important proprieties compared with the folded plate structures. These capacities give a wide degree of transformability, while maintaining suitable mechanical performances in all the possible configurations. Because of the complexity, the experimentations on these themes are often connected with various engineering branches (aerospace, electronics, ...) to coordinate aspects of materials chemistry, mechanical performances, architectural composition and building production. Besides, the kinematics required for this kind of object involves the phenomenon of wearing and tearing of the components, which ineluctably reduces its life cycle.

In recent times, light materials, like wood, glass, metallic plates and plastics of last generation, a result of technological innovations, are being used

7. Herbert Bayer et al., Albers J., Concernig fundamental design, in Bauhaus 1919-1928 (Boston: Branford, 1952). 
more and more. Thanks to this, the morphological, aesthetical aspect of the folded plate structures is exalted, guaranteeing extreme lightness to the general structure.

These characteristics make the foldable surfaces suitable for applications in building types that need these performances: environmental emergencies, worldly and cultural events, temporary houses for migrants, protection of the cultural heritage. In these sectors, in fact, it is possible to exploit full lightness and reversibility performances of this type of structures, to reduce the costs of transport and the time of installation, besides exploiting the strong communicative ability of the employed geometries.

Moreover, applications with similar results are those tied up to the adaptability and the responsivity of the surfaces, addressed to the theme of environmental sustainability. This approach leads to the so called "active building". In this way the possibility of having building envelops able to modify their own geometry in relationship with climatic conditions, is one of the challenges that the world of architecture is aiming at in the last decades, by looking for a compromise between sophisticated technology and the economic sustainability of the projects.

\section{Geometry}

The geometry that controls the foldable surfaces is related to the Oriental Art of Origami, in which the main structuring element is the fold. Folds allow kinematism and, at the same time, make the element more rigid. In particular, putting the XY reference system on the horizontal plane, it is possible to distinguish the mountain folds and the valley ones. The first ones tend to move, while closing, in the positive direction of the $\mathrm{Z}$ axis, the other ones move in the opposite direction. Besides these, there is a different type of fold, called the reverse fold $^{8}$ that is fundamental in the concept of origami. ${ }^{9}$. These are transversal with respect to the principle ones that are inverted because of them. In fact a mountain fold changes itself in a valley one if it is crossed by a reverse fold, and vice versa. It must be considered that the reverse folds maintain their characteristics, as mountain or valley folds, without any change.

The geometric relationship between the different types of folds is determined by three angles (Figure 9): the $\alpha$ angle (fixed, acute and determined by the main fold and the reverse fold); the inflection $\Phi$ angle; the $\delta$ angle (the opening between the main folds). If $\Phi=180^{\circ}$ and $\delta=180^{\circ}$, the surface lies on the plane; if $\delta=0^{\circ}$ and $\Phi=180^{\circ}-2 \alpha$, it is completely closed ${ }^{10}$.

8. Andrea Casale and Graziano M. Valenti, Architettura delle superfici piegate. Le geometrie che muovono gli origami (Roma: Ed. Kappa, 2012).

9. Peter Engel, Folding the Universe: Origami from Angelfish to Zen (New York: Vintage Books, 1999).

10. Hani Buri and Yves Weinand, “ORIGAMI-folded plate structures, architecture," pres. at the $10^{\text {th }}$ World Conference on Timber Engineering (Miyazaki, Japan, 2-5 June 2008) http://bit.ly/1TEgMPR. 


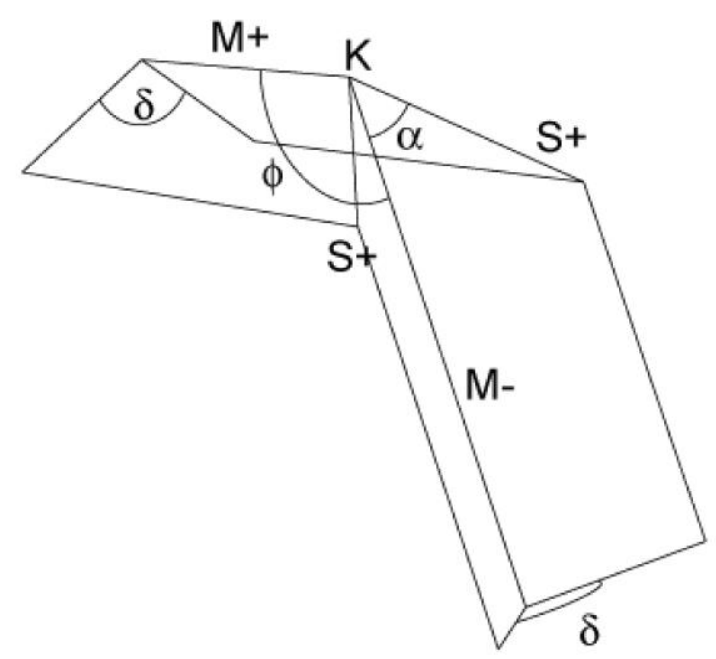

Figure 9. Reverse Folde $e^{3}$

The dislocation of the folds generates different kinds of configurations: ${ }^{11}$

- An irregular tessellation makes a complex and hardly controllable surface;

- A tessellation with polygons different from each other, leads to a specific and fixed configuration;

- If the subdivision of the surface has equal groups of tassels then it allows getting a structured, variable configuration.

This third type of folded surfaces probably represents the most interesting among architectonic or engineering applications; in particular the most recurrent are two; they are respectively called Yoshimura and Miura Ori.

The Yoshimura pattern ${ }^{12}$ (also called Diamond pattern) is characterized by a series of longitudinal folds, crossed by counterposed reverse folds that make continual inversions between the mountain and the valley folds (Figure 10). The related tessellation tends to close the surface on itself; its geometry is similar to the cylinder one on the base of the distribution of the reverse folds, the tassels can be triangular or quadrilateral. In this second case the preferential direction of the movement of the foldable surface is rectilinear. The second type of pattern, with triangular tassels, gives to the surface other degrees of freedom, in addition to the possibility of movement along a line. The Miura Ori pattern $^{13}$ (or Herringbone Pattern) is characterized by the repetition of the longitudinal folds, crossed by reverse folds (Figure 11). But in this case the last ones are concordant. This kind of tessellation makes a corrugation of the

11. Andrea Casale and Michele Calvano, "House of cards. The fold for the construction of articulated surfaces," DISEGNARECON 9 (2012): 289-300.

12. Yoshimura, Yoshimaru, "On the mechanism of buckling of a circular cylindrical shell under axial compression," in NACA Technical Memorandum 1390 (1955).

13. Koryo Miura, "Folding a plane-scenes from nature technology and art," in Symmetry of structure, interdisciplinary Symposium, Aug 13-19 (Budapest: Danvas and Nagy, 1989). 
surfaces, even if the surface remains along a plane. The tassels are quadrilateral and the movement imposed to one of them has an effect on the configuration of the surface. The single quadrangle is defined by the dimensions a and $b$ of $\gamma$ angle; its kinematism is defined by the dihedral angle $\theta[0, \pi / 2]$, formed by the tassel and the horizontal plane xy. ${ }^{14}$ The other parameters depend on them, so defining the geometry in all possible configurations (Figure 12).

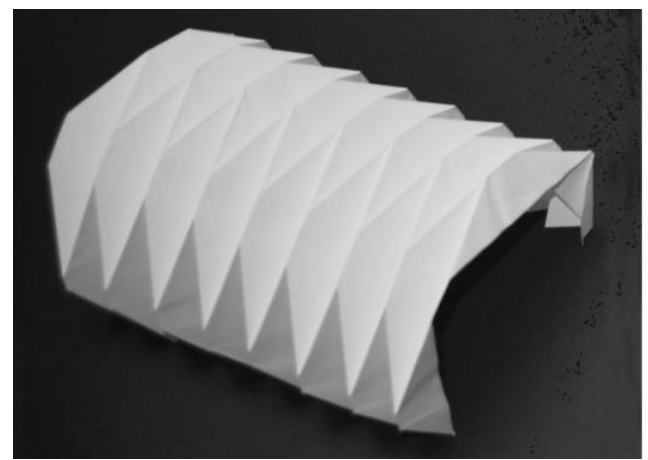

Figure 10. Yoshimura Pattern
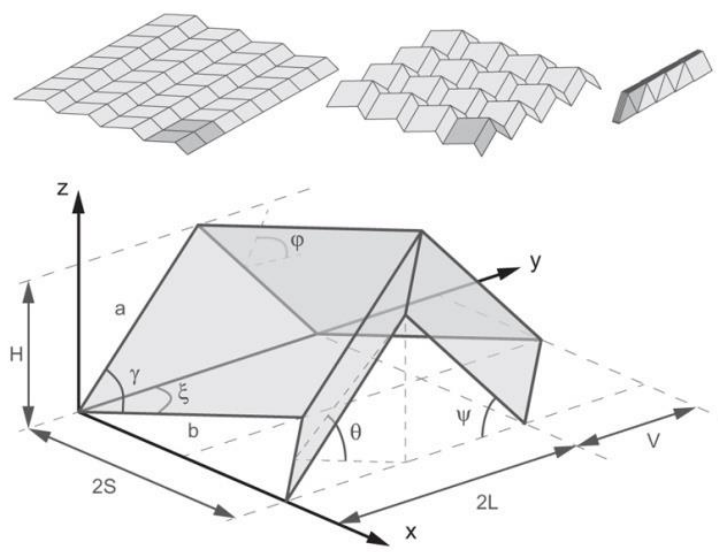

Figure 12. Miura Ori Unit Cell ${ }^{1}$

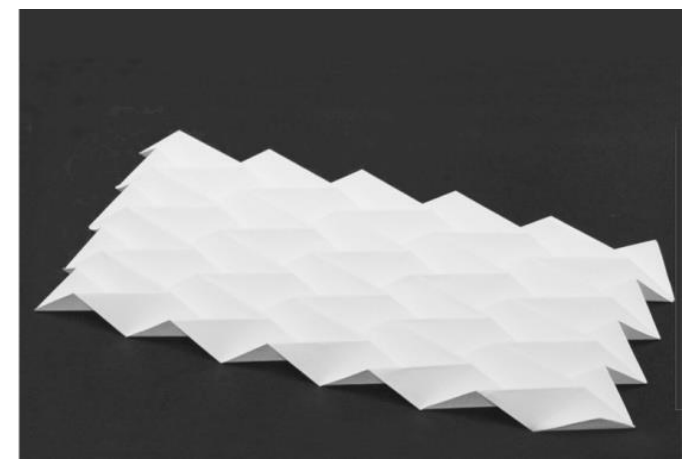

Figure 11. Miura Ori Pattern

$$
\begin{aligned}
& \mathrm{H}=\mathrm{a} \cdot \sin \theta \sin \gamma \\
& S=b \times \frac{\cos \theta \operatorname{os} \theta t}{\sqrt{1+\cos ^{2} \theta \tan ^{2} \gamma}} \\
& L=a \times \sqrt{1-\sin ^{2} \theta \sin ^{2} \gamma} \\
& V=b \times \frac{1}{\sqrt{1+\cos ^{2} \theta \tan ^{2} \gamma}}
\end{aligned}
$$

\section{Innovative Applications and Materials}

The project KREO is inserted in this line of research. It is developed in the Department of Civil Engineering and Architecture (DICAR) of the University of Catania and in the Istitute for Polymers, Composites and Biomaterials (IPCB) of the National Research Council of Italy (CNR) - Section of Catania. KREO, acronym of Kinetic, Responsive Envelop by Origami, is the prototype, at an experimental stage, of a kinetic foldable building component, potentially usable for various purposes. In the first experimentation (Figure 13) it will be optimized for the coverage of archaeological areas so as to guarantee its

14. Mark Schenk and Simon D. Guest, "Geometry of Miura-folded metamaterials," in Proceedings of the National Academy of Sciences 110 (2013): 3276-3281. http://www.pnas. org/cgi/doi/10.1073/pnas.1217998110. 
fruition in case of unfavorable climatic conditions (precipitations or excess sunshine).

This decision is motivated from the great diffusion of cultural heritage in southern Italy and in the Mediterranean area. Also, both excavation phase, to search for artifacts, and then the fruition of sites, could take place even during the extremely warm hours of the summer days. This could encourage the archeological tourism of the areas, thanks to better comfort conditions.

When it is unnecessary, it can be completely closed again, packaged so minimizing the visual impact on the cultural heritage. It is clear that, once debugging the technology, it will be possible to experiment other uses of it, for expositions, for temporary shelters and other uses. The flexibility of employment will increase the investors' desirability, once the starting phase is over and marketing is going on.

In this first step, the main performances of KREO and of its components have been defined, as it will be shown.

- Transportability is not expressed not only by lightweight. Looking closely at the performance, it is composed by packaging and modularity. The first one concerns the possibility of compacting the structure and reducing its volume; the second one is concerned with the possibility of assembling some elements to get a structure of great ampleness.

- Assemblability concerns the rapidity of assemblage or rather the facility of assembling its components. Performance that extends up to the limit of self-building. This is not a chimera if the structure is endowed with mechanisms that make it possible. Moreover, the low weight minimizes the stress of the base material. In addition to the lightness of the employed components, the necessity of intermediary elements of support or the type of fixing among the various parts must be considered. It is clear that, to proceed with dry assembly, it is essential to study with care the technology of junction to get isolation from air and water.

- A further specification of these requisites is reusability. The possibility of reusing the object reduces the cost of production and management, but a careful choice of suitable materials and technologies is necessary.

- In the recent past the flexibility of the structure was considered the ability to widen the covered surface by assembling some elements, in order to get the so-called modularity that we have already mentioned. Today the previous concept has changed into responsivity that is the ability of the object to suit the required needs. This performance can be achieved by the possibility of changing form. This characteristic can be useful to correct the screening effect of the solar radiation, the disposal of rain, the acoustic answer, the transmittance of the envelope and so on.

- Looking at the lightweight of the shell, it is possible to notice the elevated level of sustainability that it must possess. In the last years this 
term has assumed a value exclusively addressed to the energetic saving. A "cool" meaning that has partly circumscribed the original one, to which instead this kind of architectures necessarily owes to extend.

Resolving in a single object the different performances listed here, the architecture of small staircases is a good test, thanks to the minimization of the use of materials and energy. The pavillion, prototype for excellence, is therefore the highest expression of experimental design.

Our prototype is made by a creased sheet of composite material. The mesh of tessellation is studied so as to guarantee the folding and to increase besides the mechanical performances, thanks to the increase of the rigidity of the form.

We are firstly testing two composite materials that have a thermoplastic elastomer (TPE) matrix and a carbon fiber (CF) or hemp fabric reinforcement. These materials should ensure the possibility of realizing a pre-folded surface with a good capacity to be completely closed or opened.

The prototype is able to answer the needs in input, thanks to the ability of modifying its own configuration, lightweight, the low cost of the component, etc. KREO will be articulated in three phases: the geometric modeling, to investigate on the employable textures (triangular, quadrangular, rectangular, ...); the evaluation of the effects of the tessellation on kinematic (investigating on the degrees of movement); the application of the manufactured article in architecture (through physical models in various staircases, and parametric).

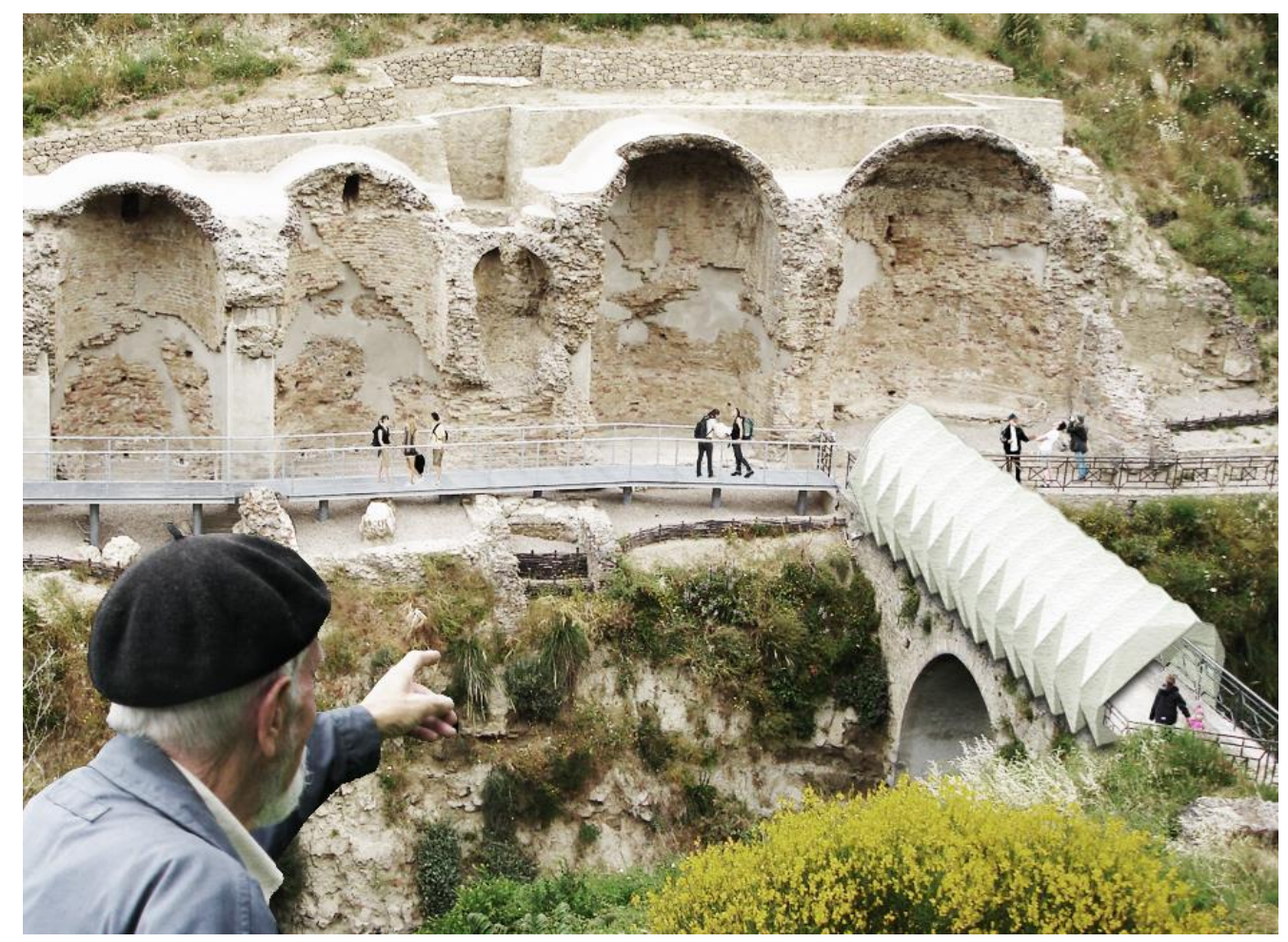

Figure 13. Simulation - KREO on a Cultural Heritage 


\section{Conclusions}

Kinetic architectures are particular types of constructions designed as mechanisms. Foldable architectures are the most promising among them. The movement allows the building components to achieve a large number of properties (flexibility, reusing, lightness, ...). Thanks to them these kinds of constructions are particularly suitable to give an answer to the most complex needs of a contemporary society.

The KREO project that has been developed by a group led by Catania's University, is addressed to the design of a particular folded architecture, useful as shelter for cultural heritage, minimizing their exposition to the environmental risks. This paper deals with the first steps of the research; it is about the definition of the state of art, the characteristics of base materials and possible applications.

\section{Bibliography}

Bayer, Herbert et al. Albers J., Concernig fundamental design, in Bauhaus 1919-1928. Boston: Branford, 1952.

Buri, Hani and Yves Weinand. "ORIGAMI-folded plate structures, architecture." Presented at the $10^{\text {th }}$ World Conference on Timber Engineering. Miyazaki, Japan: 2-5 June 2008. http://bit.ly/1TEgMPR.

Casale, Andrea and Graziano M. Valenti. Architettura delle superfici piegate. Le geometrie che muovono gli origami [Architecture of folded surfaces. Geometries that move the origami]. Roma: Ed. Kappa, 2012.

Casale, Andrea and Michele Calvano. "House of cards. The fold for the construction of articulated surfaces." DISEGNARECON 9 (2012): 289-300.

Engel, Peter. Folding the Universe: Origami from Angelfish to Zen. New York: Vintage Books, 1999.

Loonen, Roel C. G. M. et al. "Climate adaptive building shells: State-of-the-art and future challenges." Renewable and Sustainable Energy Reviews 25 (2013): 483493.

Miura, Koryo. "Folding a plane-scenes from nature technology and art." In Symmetry of structure, interdisciplinary Symposium, Aug 13-19. Budapest: Danvas and Nagy, 1989.

Otto, Frei. ILEK/IL5. Stuttgart: ILEK press, 1972.

Schenk, Mark and Simon D. Guest. "Geometry of Miura-folded metamaterials." In Proceedings of the National Academy of Sciences 110 (2013): 3276-3281. http://www.pnas.org/cgi/doi/10.1073/pnas.1217998110.

Yoshimura, Yoshimaru. "On the mechanism of buckling of a circular cylindrical shell under axial compression." In NACA Technical Memorandum 1390 (1955). 
